Devenir, volume 19, numéro 2, 2007, pp. 109-130.

\title{
CLASSIFICATION DIAGNOSTIQUE 0-3 ANS RÉVISÉE : UNE NOUVELLE PRÉSENTATION DES TROUBLES DE LA RÉGULATION DU TRAITEMENT DES STIMULI SENSORIELS
}

\author{
Diagnostic Classification DC:O-3R : a new presentation of the \\ Regulation Disorders of Sensory Processing
}

Jean-Marc Scholl ${ }^{1}$

\section{Introduction}

En 1994 est parue la première «Classification Diagnostique des Troubles de la Santé Mentale et du Développement de la Petite Enfance » (DC:0-3). Depuis lors, elle a reçu une reconnaissance internationale et des études de validité ont démontré sa fiabilité (Guedeney N., Guedeney A., Danon et coll., 2002). En août 2005 est parue l'Édition Révisée de la Classification (DC:0-3R) dont la traduction en français est en cours. Le diagnostic de Trouble de la Régulation est certainement le plus original et le plus innovateur de cette Classification ; il ouvre le praticien à un sens clinique plus fin et donne accès à des observations qui enrichissent la pratique psychothérapeutique (Thomas \& Harmon, 1998). Dans cet article, après un bref aperçu de l'origine du concept des troubles de la régulation, nous décrirons la nouvelle présentation des tableaux cliniques des Troubles de la Régulation du traitement des stimuli sensoriels de la Classification Diagnostique Révisée puis nous indiquerons ce qui leur est spécifique par rapport à l'édition précédente. Enfin, nous évoquerons notre expérience quant à leur utilisation clinique.

\section{L'origine du concept des troubles de la régulation}

Dès 1972, Jean Ayres a développé le concept "d'intégration sensorielle" en lien avec des difficultés d'apprentissage du petit enfant (Ayres, 1972). Winnie Dunn, psychologue du développement, a décrit l'éventail clinique du "profil sensoriel" des enfants ordinaires avec une validation développementale (Sensory Profile, 1999) ; elle relève aussi les conséquences - dès le plus jeune âge - liées aux difficultés d'intégration sensorielle. La capacité à traiter et intégrer les informations sensorielles influence des spécificités du fonctionnement du bébé et de l'enfant (Greenspan, 1992). Un lien existe entre ces capacités et la notion de tempérament (Chess \& Thomas, 1986 ; Sturm, 2004), et Brazelton avait déjà souligné en 1969 des

\footnotetext{
${ }^{1}$ Scholl Jean-Marc, Service de Santé Mentale, rue des Déportés, 30 ; 4800 Verviers ; Belgique Jean-Marc.Scholl@chu.ulg.ac.be

L'auteur a traduit le texte original de la Classification Révisée en essayant de restituer la polysémie des sens cliniques de chaque mot anglais. Cette traduction ne présage pas de la traduction officielle de la Classification Révisée à paraître en français.

Cet article fait partie d'une recherche et d'un travail de thèse de doctorat réalisés sous la tutelle de l'Université de Liège (Professeurs Marc Ansseau et Francis Pérée). J'adresse mes remerciements au Dr Paule Philippe pour les échanges cliniques qui ont alimenté la réflexion et l'expérience. Je remercie également le travail des lecteurs anonymes dont les commentaires m'ont aidé à rédiger la version actuelle de cet article.
} 
caractéristiques et compétences propres du bébé. Dès 1972, Alexander Thomas et Stella Chess ont décrit l'ajustement nécessaire entre les capacités d'intégration sensorielle de l'enfant et les demandes sociales qui lui sont adressées au sein de son milieu physique, ajustement qui lui permet un bon apprentissage et un bon développement et qui, lorsqu'il n'est pas respecté, va altérer ses capacités d'intégration sensorielle. Le bon équilibre de cet ajustement, que ces auteurs ont nommé le "goodness-of-fit", est lui-même fonction de chaque enfant et spécifique des caractéristiques de son tempérament (Chess \& Thomas, 1986 ; Williamson \& Anzalone, 2001). Différents auteurs comme Mary Rothbart ont montrés ensuite que de bonnes capacités de régulation de l'intégration sensorielle font intervenir des mécanismes neurophysiologiques régulateurs (Rothbart, Chew, Gartstein, 2001). Rappelons encore que Daniel Stern, à partir de l'observation du nourrisson non pathologique, avait aussi mis en évidence des capacités innées et propres à chaque enfant, capacités qui interviennent dans les processus d'attachement et d'intersubjectivité.

Par ailleurs, en 1977 des cliniciens américains de la petite enfance fondèrent, avec un philanthrope, Irving Harris, une organisation non-gouvernementale et indépendante, pour promouvoir les échanges entre les cliniciens ainsi que les recherches sur les connaissances récentes concernant le développement précoce de l'enfant. Parmi les membres fondateurs on trouve Robert Emde, élève de Spitz, Stanley Greenspan, Kathryn Barnard, Sally Provence, Alicia Lieberman, Joy Osofsky, Arnold Sameroff, Albert Solnit, Réginald Lourie, Charles Zeanah. Cette organisation se nommera le Zero to Three National Center for Infants, Toddler and Families (NCITF). Au sein de cette organisation, en 1987, la présidente de l'époque, Kathryn Barnard, fonda un groupe de travail chargé d'élaborer une classification diagnostique spécifique pour la petite enfance. Ce groupe pluridisciplinaire avait la volonté de proposer des premières catégories cliniques provisoires en attente de recherches et de révisions ultérieures. Elles furent réalisées en rassemblant les observations cliniques et en cherchant à mieux identifier une psychopathologie. Établir une classification diagnostique du bébé et du jeune enfant nécessitait de définir des tableaux cliniques ; cela impliquait la recherche d'une meilleure connaissance des symptômes, de leur expression en fonction de l'âge du développement, de définir objectivement un seuil au-delà duquel la manifestation devient un symptôme et non plus seulement une variation de la normale. La Classification 0-3 ans (« Classification Diagnostique des Troubles de la Santé Mentale et du Développement de la Petite Enfance, DC:0-3) fut publiée en 1994 et connu rapidement un essor international puisqu'elle est traduite ou en cours de traduction en 14 langues. Elle veut être une simple photographie de l'instant présent, toujours susceptible de modifications et d'évolution. La classification est multi-axiale, relationnelle, souple, dynamique pour la connaissance et le traitement des nourrissons, des jeunes enfants et de leur famille (Thomas \& Harmon 1998). Dans la Classification 0-3 ans, les Troubles de la Regulation sont un des diagnostics possibles parmi les diagnostics primaires de l'axe I. Au sein du groupe des cliniciens, c'est particulièrement Greenspan et DeGangi qui ont réalisé la recherche et l'élaboration des tableaux cliniques des Troubles de la Régulation (Greenspan 1992; Greenspan \& Wieder 1993; DeGangi et coll.1991, 1993, 1995, 1999, 2000). Dans la Classification, ces troubles sont caractérisés par les difficultés du bébé ou du jeune enfant à réguler ses comportements et ses processus physiologiques sensoriels, d'attention, moteurs ou émotionnels et à maintenir un état de calme, de vigilance ou un état émotionnel positif (DC:0-3, p.41, 1994). Dans l'axe I, on rencontre également le diagnostic de Trouble touchant de Multiples Domaines du Développement (MSDD, Multisystem Developmental Disorder) dans lequel sont décrits des troubles sévères d'intégration sensorielle ; ce diagnostic correspond au champ clinique du spectre autistique.

Le concept de Troubles de la Régulation est cependant critiqué par certains auteurs tels que Angols et Egger qui voudraient remplacer cette entité clinique par des expressions précoces 
des diagnostics classiques du DSM IV (TADA : hyperactivité et/ou déficit d'attention, TED : Troubles Envahissants du Développement, TOP : Trouble oppositionnel avec provocation). Pourtant, différentes études démontrent la fiabilité de la Classification 0-3 ans (DC:0-3) ; elle présente une plus grande fiabilité entre les évaluateurs ainsi que pour le test-retest lorsqu'elle est comparée à la classification CIM-10/ICD-10 (Skovgaard, Houmann, Landorph et coll., 2004; Skovgaard, Houmann, Christiansen et coll., 2005).

La première Classification 0-3 ans de 1994 avec ses catégories provisoires vient de connaître une révision en 2005. Les tableaux cliniques des Troubles de la Régulation ont été affinés et sont présentés dans cet article.

\section{Présentation des Troubles de la Régulation dans l'Édition Révisée (DC:0-3R, 2005)}

\section{A) Les différentes modalités des Troubles de la Régulation}

La nouvelle Classification « Diagnostic Classification of Mental Health and Developmental Disorders of Infancy and Early Childhood: REVISED EDITION » : « DC:0-3R », 2005, donnent trois modalités de Troubles de la Régulation du traitement des stimuli sensoriels (Regulation Disorders of Sensory Processing) :

1. Hypersensible

2. Hyposensible/Sous-réactif

3. Recherchant des stimulations sensorielles et/ou Impulsif

La modalité Hypersensible est elle-même subdivisée en deux types :

Type A: Craintif/Prudent

Type B: Opposant/Provoquant

Ces Troubles de la Régulation sont inclus dans l'axe I des diagnostics primaires, dans la catégorie 400 qui en précise plusieurs tableaux cliniques avec les références suivantes :

Axe I

400. Trouble de la Régulation du traitement des stimuli sensoriels

410 : Hypersensible

411 : Type A: Craintif/Prudent

412 : Type B: Opposant/Provoquant

420 : Hyposensible/Sous-réactif

430 : Recherchant des stimulations sensorielles et/ou Impulsif

Avant d'aborder les différents tableaux cliniques, la Classification décrit les répercussions, dans la vie au quotidien de l'enfant, des difficultés qu'il présente à traiter les stimuli sensoriels.

\section{B) Le retentissement chez l'enfant de ses difficultés à traiter les stimuli sensoriels}

Les Troubles de la Régulation du traitement des stimuli sensoriels sont constitutionnellement déterminés par les réponses de ces enfants aux stimuli sensoriels. Le diagnostic de Trouble de la Régulation du traitement des stimuli sensoriels se réfère aux difficultés de ces enfants à

réguler : - leurs émotions

- leurs comportements

- leurs habilités motrices

en réponse aux stimulations sensorielles.

Ces difficultés conduisent ces enfants à un moins bon développement et un moins bon fonctionnement dans leurs aptitudes. Les nourrissons et les jeunes enfants développent des 
«patterns d'attitudes» qui affectent leur fonctionnement au quotidien, notamment leurs interactions avec les adultes et les autres enfants.

Les stimuli sensoriels comprennent le toucher, la vue, les bruits, le goût, l'odorat, les sensations de mouvements dans l'espace, et la conscience de la position du corps dans l'espace. Chaque enfant a une modalité spécifique pour répondre aux stimuli sensoriels de l'environnement. Certains enfants ont des difficultés $\left(1^{\circ}\right)$ au niveau du traitement des stimuli sensoriels entrants et $\left(2^{\circ}\right)$ au niveau de la régulation des réponses qu'ils apportent à ces stimuli. Ces difficultés à traiter les stimuli et à réguler leurs réactions peuvent interférer avec l'ensemble du développement social et émotionnel de l'enfant et avec ses habilités motrices, plus particulièrement avec l'habilité de l'enfant à participer aux activités appropriées à son âge.

Les personnes qui s'occupent de l'enfant ("caregivers"), par leurs interventions, régulent et tempèrent les réponses comportementales de l'enfant aux stimuli sensoriels. Ces personnes ("caregivers") qui modèrent ou sollicitent les patterns comportementaux de l'enfant peuvent améliorer les difficultés de régulation de l'enfant. En sens inverse, la méprise et le décalage entre la réponse constitutionnelle de l'enfant aux stimuli sensoriels et les réactions des personnes qui s'occupent de l'enfant peuvent intensifier de telles difficultés de régulation. Ces difficultés peuvent se mêler à d'autres. Les Troubles de la Régulation du traitement des stimuli sensoriels peuvent coexister avec d'autres troubles, par exemple un Trouble de l'Anxiété $^{(D C: 0-3 R, p .29)}$ (Cordeiro \& Caldeira da Silva, 1998).

Pour chaque modalité de Trouble de la Régulation du traitement des stimuli sensoriels, des réactions spécifiques à ces stimuli apparaissent chez les enfants. Les nourrissons et les enfants Hypersensibles aux stimuli sensoriels les expérimentent comme désagréables et ils les évitent. Les enfants Hyposensibles/Sous-Réactifs ont besoin d'une forte intensité d'entrée sensorielle avant de pouvoir y répondre. Les enfants Recherchant les stimulations sensorielles et/ou Impulsifs cherchent activement à satisfaire leurs besoins d'entrées sensorielles de haut niveau. En préalable à la description de chacun des tableaux cliniques, la Classification décrit les réactions aux stimulations sensorielles chez ces enfants. Abordons maintenant la description de ces réactions et de ces tableaux.

\section{C) Le diagnostic des différents tableaux cliniques des Troubles de la Régulation}

Chacun des tableaux cliniques est décrit en trois volets :

1. Les patterns de la réactivité sensorielle. Ils décrivent les difficultés du traitement des stimuli sensoriels.

2. Les patterns moteurs. Ils décrivent les difficultés motrices.

3. Les "patterns d'attitudes". Ils décrivent les attitudes et comportements spécifiques. L'enfant doit correspondre à l'allure générale d'un tableau sans devoir correspondre strictement à tous les items de ce tableau.

\section{0 : Hypersensible ${ }^{(D C: 0-3 R, p .29)}$}

Cette catégorie 410 n'est qu'une description du "pattern hypersensible" à des stimuli variés qui est commun et sous-jacent à deux types de "patterns d'attitudes" différents :

- Type A : Craintif/Prudent (411)

ou

- Type B : Opposant/Provoquant (412)

C'est le choix de l'un de ces deux types qui établit le réel diagnostic Hypersensible (la catégorie 410 est insuffisante à elle seule). 
Les nourrissons et les jeunes enfants qui sont hypersensibles aux stimuli sensoriels les expérimentent comme désagréables voire aversifs. Les stimuli qui fréquemment déclenchent des conduites d'évitement comprennent un léger toucher, des bruits forts, des lumières brillantes, des odeurs et des goûts peu connus, des textures rêches, et/ou des sensations de mouvements. Ces enfants évitent les stimuli sensoriels ou montrent des réactions d'aversion à leur égard. Les enfants hypersensibles :

- ont des difficultés à moduler leurs réponses aux stimuli

- $\quad$ sont vite accablés par les stimuli sensoriels qui font partie de la vie quotidienne

- ont tendance à connaître un stress considérable lorsqu'ils essaient de gérer leurs intenses réponses à ces stimuli.

Les réponses comportementales à divers stimuli pourront varier suivant :

$1^{\circ}$ ) l'intensité, la durée ou la localisation des stimuli (par exemple, un enfant tolérera un stimulus unique mais il répondra en s'opposant ou avec de l'aversion à des stimuli subséquents du même type)

$2^{\circ}$ ) le niveau d'éveil d'un enfant (par exemple, une enfant sera calme et en alerte dans la matinée mais elle deviendra surexcitée quand elle sera fatiguée en fin de journée)

$3^{\circ}$ ) la source du stimulus (par exemple, un enfant répondra différemment à un toucher selon qu'il en a l'initiative ou qu'il lui est imposé)

Il est aussi important de noter que le type A: Hypersensible Craintif/Prudent peut se rencontrer avec un Trouble d'Anxiété, et que le type B: Hypersensible Opposant/Provoquant peut se rencontrer avec des troubles du comportement tel qu'un Trouble oppositionnel avec provocation.

\section{1 : Type A: Craintif/Prudent ${ }^{(D C: 0-3 R, p .30)}$}

1. Les patterns de la réactivité sensorielle

(a) Ces patterns sont caractérisés par une hyper-réactivité aux stimuli sensoriels, comme un léger toucher, des bruits forts, des lumières brillantes, des odeurs et des goûts peu connus, des textures rêches, et/ou des sensations de mouvement.

Exemples : Un petit enfant n'est pas capable de tolérer des jeux de bousculade ou le chahut ou des jeux à bascule. Un autre enfant pourra donner un signal de détresse quand il est placé dans une position couchée ou lors des changements de position (particulièrement si la tête est inclinée en arrière).

(b) Les réponses aux stimuli sensoriels peuvent comprendre :

- $\quad$ Craintes - appréhensions

- $\quad$ Pleurs - cris

- $\quad$ "Givré" - bloqué

- Tentatives d'échapper au stimulus

- Distractibilité accrue

- $\quad$ Accès de colère - "faire des caprices"

- Réactions excessives d'alarme, de sursauts, de peur

- $\quad$ Agitation motrice

- Réduction de la tolérance face à une variété d'aliments, de textures, de goûts et d'odeurs.

2. Les patterns moteurs

L'hypersensibilité et l'aversion aux stimuli sensoriels pourront limiter l'enfant dans ses expériences de manipulations ou d'interactions avec l'environnement, conduisant à un déficit fonctionnel dans le développement moteur. Les patterns moteurs, variables selon les enfants, comprennent :

(a) Des difficultés de contrôle postural et du tonus 
(b) Des difficultés de coordination motrice fine (souvent associées - chez les enfants hypersensibles - à leur moindre ouverture à expérimenter les jeux, jouets et autres objets).

(c) Des difficultés de planification motrice

(d) Une moindre exploration de l'environnement que celle attendue pour l'âge

(e) Une limitation face aux jeux sensori-moteurs

3. Les "patterns d'attitudes"

Les "patterns d'attitudes" du nourrisson et du jeune enfant présentant le Type A:

Hypersensible Craintif/Prudent comprennent une prudence excessive, de l'inhibition, de la crainte et de l'appréhension. En plus de ces patterns, les attitudes des nourrissons et des petits-enfants présentant un Type A: Hypersensibles peuvent inclure :

(a) Inquiétudes ou peurs excessives

(b) Timidité à répondre à de nouvelles personnes, timidité lorsque l'enfant est confronté à des lieux nouveaux, à de nouveaux objets

(c) Distractibilité par des stimuli sensoriels

(d) Impulsivité lorsqu'il est "surchargé" de stimuli sensoriels

(e) De fréquents moments d'irritabilité et de pleurs

(f) Capacité limitée à s'apaiser ("d'auto-apaisement") (par exemple, après s'être réveillé difficultés à se rendormir)

(g) Difficultés à quitter l'état émotionnel lié à des frustrations ou des déceptions

(h) Évitement ou peu d'engagement dans de nouvelles expériences ou de nouvelles sensations

N.B. Tendance à être effrayé et à l'agrippement dans les situations nouvelles ${ }^{(D C: 0-3, p .47)}$

\section{2 : Type B: Opposant/Provoquant ${ }^{(D C: 0-3 R, p .32)}$}

1. Les patterns de la réactivité sensorielle sont identiques à ceux du Type A: Hypersensible Craintif/Prudent

2. Les patterns moteurs sont aussi identiques à ceux décrits dans le Type $\mathrm{A}$

3. Les "patterns d'attitudes" dans le Type B sont différents de ceux du Type A.

Les enfants Hypersensibles Opposants/Provoquants ont tendance à éviter de nouvelles expériences ou à peu s'y engager et, en général, ils ne sont agressifs que lorsqu'on les provoque. Les "patterns d'attitudes" des nourrissons et des jeunes enfants présentant le Type B: Hypersensible Opposant/Provoquant comprennent :

(a) Des comportements négatifs (par exemple, chez les jeunes enfants : faire beaucoup d'embarras pour des futilités ; chez l'enfant en âge préscolaire : faire de fréquentes réflexions négatives face aux demandes parentales ou avoir des accès d'irritation, voire des colères)

N.B. Les nourrissons ont tendance à être exigeants et difficiles ${ }^{(D C: 0-3, p .46)}$

(b) Garder la maîtrise

(c) Défi (adoptant la conduite opposée à ce qui est demandé ou attendu)

(d) Préfère les répétitions, les absences de changements, et si des changements sont nécessaires, l'enfant procède lentement et progressivement

(e) Difficultés à s'adapter à des changements concernant la routine ou des plans préétablis

(f) Compulsions et perfectionnisme

(g) Évitement ou peu d'engagement dans de nouvelles expériences ou de nouvelles sensations

N.B. À d'autres moments ces mêmes enfants peuvent être gais et dociles ${ }^{(D C: 0-3, p .46)}$ 


\section{0 : Hyposensible/Sous-réactif ${ }^{(D C: 0-3 R, p .32)}$}

Les nourrissons et les jeunes enfants hyposensibles ont besoin d'une forte intensité d'entrée sensorielle avant de pouvoir y répondre. Ces enfants sous-réactifs sont généralement calmes et attentifs. Ils semblent souvent sous-réactifs à leur environnement et peu réactifs aux propositions venant d'autres personnes. Des efforts importants ou persévérants peuvent être nécessaires pour engager la relation avec un enfant sous-réactif. Bien que les enfants sousréactifs peuvent apparaître tristes ou désintéressés à leur environnement, leur repli et leur manque de réceptivité reflètent habituellement leur insuffisance à atteindre le seuil d'éveil et d'excitabilité qui pourrait les motiver à agir et à interagir.

En considérant le diagnostic de Trouble de la Régulation Hyposensible/Sous-réactif, le clinicien doit être attentif à déterminer $\left(1^{\circ}\right)$ que la limitation de la réponse sociale de l'enfant ne reflète pas une diminution de l'engagement social caractéristique d'un Trouble Envahissant du Développement, $\left(2^{\circ}\right)$ que le repli sur soi de l'enfant ne reflète pas une humeur dépressive comme une Dépression Majeure ou un Trouble dépressif, et $\left(3^{\circ}\right)$ que le retrait de l'enfant n'est pas un symptôme d'un Trouble de l'Anxiété tel qu'un Trouble de l'Anxiété Sociale.

\section{Les patterns de la réactivité sensorielle}

(a) Sous-réactivité aux sons, mouvements, odeurs, goûts, toucher et aux stimuli proprioceptifs

(b) Pour le nourrisson : un manque de réceptivité/réponses aux sensations et aux propositions sociales qui lui sont faites

2. Les patterns moteurs

(a) Exploration limitée

(b) Éventail de jeu limité

(c) Recherche d'entrées sensorielles spécifiques, souvent trouvées dans des activités répétitives et sensorielles telles que balancements, sauts, monter et descendre du lit.

(d) Nonchalant - mou

(e) Difficultés de planification motrice et maladresses dues à une pauvreté du développement du schéma corporel - conséquence d'une sous-réactivité aux stimuli tactiles et proprioceptifs

3. Les "patterns d'attitudes"

(a) Manque apparent d'intérêt à l'exploration des propriétés d'objets, jouets, jeux stimulants, ou à s'engager dans des interactions sociales

(b) Apparence apathique

(c) Fatigabilité

(d) Retrait face aux stimuli

(e) De l'inattention

En plus des symptômes ci-dessus, les nourrissons et les jeunes enfants avec un Trouble de la Régulation Hyposensible/Sous-réactif peuvent apparaître retardés ou déprimés. Les enfants en âge préscolaire avec ces troubles peuvent être "en désaccordage" relationnel dans les conversations et ils peuvent avoir un éventail limité d'idées et d'imagination dans les comportements quotidiens et les jeux.

\section{0 : Recherchant des stimulations sensorielles/Impulsif ${ }^{(D C: 0-3 R, p .33)}$}

Les nourrissons et les jeunes enfants sous-réactifs que nous venons de décrire, lorsqu'ils trouvent des stimulations sensorielles, nécessitent des stimuli intenses, fréquents et/ou prolongés avant de pouvoir y répondre. Au contraire des enfants sous-réactifs, les enfants d'âge préscolaire que nous décrivons ici recherchent activement à satisfaire leurs besoins d'entrées sensorielles de haut niveau, bien plus que les enfants de même niveau de 
développement. Ce pattern de réactivité sensorielle et motrice peut être associé à un Déficit d'Attention et/ou un Trouble d'Hyperactivité (DSM IV-TR, 2000), particulièrement le Type Hyperactivité et/ou Impulsivité.

1. Les patterns de la réactivité sensorielle

Un "ardent appétit" de stimuli sensoriels intenses. Ce besoin peut mener à des comportements destructeurs ou des comportements à haut risque.

2. Les patterns moteurs

(a) Grand besoin de décharges motrices

(b) Une impulsivité diffuse

(c) Propension à faire des accidents, sans être maladroit

3. Les "patterns d'attitudes"

(a) Haut niveau d'activité

(b) Recherche constante de contact avec des personnes et des objets

(c) Recherche de stimulations au travers de fortes pressions

(d) Témérité

(e) Des comportements désorganisés en réponse aux stimulations sensorielles

En plus des symptômes ci-dessus, l'enfant en âge préscolaire peut aussi être :

- Excitable

- Agressif

- Intrusif

- Audacieux et téméraire, risquant des accidents et des blessures

- Occupé par des thèmes agressifs qu'il prétend n'être que du jeu

Il n'est pas rare, pour les enfants recherchant des stimulations sensorielles, que leur besoin pressant de contact physique avec des personnes et des objets conduise à la détérioration de propriétés d'autrui, à l'intrusion dans des espaces physiques d'autres personnes, ou à frapper sans provocation apparente. Les autres enfants et les adultes peuvent se méprendre en considérant la recherche de stimulations sensorielles comme de l'agression. Une fois que d'autres réagissent agressivement envers l'enfant, celui-ci peut à son tour commencer à être agressif avec une intention explicite.

\section{D) Comorbidités des Troubles de la Régulation}

Les Troubles de la Régulation du traitement des stimuli sensoriels peuvent coexister avec d'autres troubles (par exemple, Type A: Trouble de la Régulation Hypersensible et Trouble d'Anxiété de Séparation). Les symptômes d'un Trouble de la Régulation et ceux d'autres Troubles et désordres peuvent se recouvrir à certains degrés ( $D C: 0-3 R, p .29)$.

Contrairement à la Classification DC:0-3 qui préférait l'usage d'un seul diagnostic sur l'Axe I, la Classification DC:0-3R, établie à partir d'une expérience clinique de plus de 10 ans, montre que plus d'un diagnostic primaire sur l'Axe I peut être souvent approprié. Ainsi, un Trouble de la Régulation du traitement des stimuli sensoriels et une Dépression peuvent survenir simultanément. Dans de tels cas, il serait approprié de mettre en relief les deux diagnostics primaires dans l'intention de déterminer les traitements utiles ( $D C: 0-3 R, p .10 \& 67)$.

Il est aussi important de noter que le type A: Hypersensible Craintif/Prudent peut se rencontrer avec un Trouble d'Anxiété, et que le type B: Hypersensible Opposant/Provoquant peut se rencontrer avec des troubles du comportement tel qu'un Trouble oppositionnel avec provocation $(D C: 0-3 R, p .30)$. 
Un Trouble de la Régulation Hyposensible/Sous-réactif doit être reconnu alors que l'enfant peut apparaître retardé ou déprimé. Une lenteur dans le développement du langage peut être la conséquence d'un Trouble de la Régulation Hyposensible/Sous-réactif ( $D C: 0-3 R, p .33)$. Une déficience intellectuelle ou une humeur dépressive peuvent être concomitants à un Trouble de la Régulation Hyposensible/Sous-réactif.

Les enfants "Recherchant des stimulations sensorielles et/ou Impulsif" présentent un pattern de réactivité sensorielle et motrice qui peut être associé à un Déficit d'Attention et/ou un Trouble d'Hyperactivité (DSM IV-TR), particulièrement le Type Hyperactivité et/ou Impulsivité ( $D C: 0-3 R, p .33$ ).

Les troubles du sommeil ou de l'appétit peuvent être des symptômes de Troubles de la Régulation, ou faire partie d'autres catégories de diagnostics (DC:0-3, p.43).

\section{E) Les Troubles de la Régulation et les Diagnostics Différentiels}

Le diagnostic de Trouble de la Régulation du traitement des stimuli sensoriels comprend trois aspects : $\left(1^{\circ}\right)$ les difficultés du traitement des stimuli sensoriels, $\left(2^{\circ}\right)$ des difficultés motrices, et $\left(3^{\circ}\right)$ un «pattern d'attitudes » spécifique.

Si ces trois aspects ne sont pas caractéristiques chez un enfant, le clinicien devrait considérer des alternatives dans la Classification. Par exemple :

- Des comportements d'opposition et "d'entêtement" peuvent résulter de parents contraignants voire coercitifs (Axe II : Classification de la Relation).

- Des comportements d'opposition et "d'entêtement" peuvent refléter un trouble conduisant à un comportement perturbateur tel qu'un Trouble d'Opposition (DSM IVTR, 2000) qui n'est pas obligatoirement associé à des difficultés du traitement des stimuli sensoriels.

- L'aspect Craintif tel que décrit dans le type A: Troubles de la Régulation Hypersensible Craintif/Prudent peut refléter un Trouble de l'Anxiété (DC:0-3R, p.29).

Un nourrisson irritable et réagissant trop vivement aux expériences interpersonnelles de routine, en l'absence de difficultés de traitement de l'information sensorielle et sensori-motrice clairement identifiées, peut manifester un Trouble de l'Anxiété ou de l'Humeur (DC:0-3, p.43).

En considérant le diagnostic de Trouble de la Régulation "Hyposensible"/Sous-réactif, le clinicien doit être attentif à déterminer $\left(1^{\circ}\right)$ que la limitation de la réponse sociale de l'enfant ne reflète pas une diminution de l'engagement social caractéristique d'un Trouble Envahissant du Développement, $\left(2^{\circ}\right)$ que le repli sur soi de l'enfant ne reflète pas une humeur dépressive comme une Dépression Majeure ou un Trouble dépressif, et $\left(3^{\circ}\right)$ que le retrait de l'enfant n'est pas un symptôme d'un Trouble de l'Anxiété tel qu'un Trouble de l'Anxiété Sociale (DC:0-3R, p.33).

Les situations cliniques des Troubles de la Régulation vont des plus légères au plus importantes pour arriver à la frontière des Troubles touchant de Multiples Domaines $d u$ Développement (MSDD, Multisystem Developmental Disorder). Ces derniers, pour les formes les plus sévères, se rapprochent de l'autisme infantile. Dans ces situations frontières, c'est le sens clinique qui permet de différencier un Trouble touchant de Multiples Domaines du Développement avec des traits de Troubles de la Régulation et un Trouble sévère de la Régulation avec des caractéristiques mixtes. Ces deux états seront très sensibles à des situations de stress importants de la vie. La différenciation sera fonction de l'importance des difficultés liées à la relation, au degré des troubles du langage et de la communication, et à la 
plus ou moins bonne réponse initiale aux interventions thérapeutiques. (De même, dans les Troubles touchant de Multiples Domaines du Développement, la difficulté relationnelle n'est pas considérée comme un déficit permanent et relativement figé mais comme susceptible de modifications et d'une évolution) (DC:0-3, p.128).

\section{L'APPORT DE LA NOUVELLE PRÉSENTATION DES TROUBLES DE LA RÉGULATION DU TRAITEMENT DES STIMULI SENSORIELS (2005) PAR RAPPORT À LA PREMIÈRE ÉDITION DE 1994}

\section{A) L'intitulé du diagnostic}

Le titre du diagnostic a changé : de "Trouble de la Régulation", il est devenu : "Trouble de la Régulation du traitement des stimuli sensoriels". Ce nouvel intitulé veut porter l'attention sur les difficultés du traitement des stimuli sensoriels qui caractérisent ce trouble. (DC:0-3R, p.9)

\section{B) Les tableaux cliniques}

Chaque tableau clinique est décrit par une nouvelle répartition de trois volets : $\left(1^{\circ}\right)$ les patterns de la réactivité sensorielle, $\left(2^{\circ}\right)$ les patterns moteurs, $\left(3^{\circ}\right)$ les "patterns d'attitudes", alors que précédemment les trois volets étaient : $\left(1^{\circ}\right)$ les "patterns $d^{\prime}$ attitudes", $\left(2^{\circ}\right)$ les patterns moteurs et sensoriels, $\left(3^{\circ}\right)$ les styles relationnels de soins idéaux. La nouvelle présentation - fruit d'une expérience de plus de 10 ans - a nettement gagné en précision clinique. La description commence par "les patterns de la réactivité sensorielle", en mettant d'emblée l'accent sur la spécificité de la modalité clinique et en décrivant davantage les réactions propres à l'enfant liées à sa modalité de traitement des stimuli sensoriels. De plus, cette présentation a divisé en deux parties l'ancien volet "moteurs et sensoriels" ce qui apporte davantage de distinction descriptive. L'ancien volet qui correspondait aux "styles relationnels de soins idéaux" est supprimé. De fait, dans notre expérience, il n'apportait pas de véritables informations diagnostiques car il correspondait à des considérations générales de relations idéales à proposer à l'enfant, ce que tout thérapeute averti connaît bien. Les tableaux cliniques des "patterns d'attitudes" ont été partiellement modifiés. Pour les modalités Hypersensibles, les types A: Craintif/Prudent et B: Opposant/Provoquant sont semblables à la classification précédente mais gagnent en précision. La modalité Hyposensible/Sous-Réactif voit disparaître ses deux types : "En retrait et difficile à engager" et "Absorbé en lui-même et imaginatif". De fait, dans notre expérience, ces tableaux ne correspondaient que partiellement à la description des enfants rencontrés en clinique. Cela nous obligeait à recourir fréquemment à l'ancien diagnostic : "Autre". Actuellement, la composante possible d'une imagination importante n'est plus reprise, par contre l'ensemble du tableau gagne en cohérence. Enfin, la troisième modalité a changé d'intitulé, elle se nomme : "Recherchant des stimulations sensorielles/Impulsif", alors qu'avant elle s'intitulait : "Désorganisé du point de vue moteur/Impulsif". Le point essentiel de cette modification concerne la motricité. Préalablement on a imputé à l'enfant "une médiocre organisation et planification de la motricité", actuellement on observe : "une propension à faire des accidents, sans être maladroit", attribuant les risques d'accidents et de blessures à "une impulsivité diffuse", "un grand besoin de décharges motrices", à de la "témérité", en comprenant les comportements désorganisés comme des réactions aux stimulations sensorielles. Dans notre expérience, nous partageons ce même point de vue et, si une désorganisation motrice est réellement présente, 
elle doit être comprise comme un autre diagnostic simultanément présent. De plus, le diagnostic « Autre » de l'ancienne édition a été supprimé. Il correspondait à des formes mixtes des tableaux précédents. La classification actuelle présuppose donc que les tableaux cliniques correspondront davantage aux enfants rencontrés dans nos pratiques. Nous voulons toutefois attirer l'attention sur le fait qu'il nous semble que certains enfants peuvent présenter deux modalités simultanément. Tout particulièrement, la modalité "Recherchant des stimulations sensorielles et/ou Impulsif" pourrait se surajouter à la modalité Hypersensible. Il nous semblerait utile de pouvoir faire le diagnostic de deux modalités à la fois (peut-être avec une cotation en gradient d'intensité ou de certitude diagnostique pour chaque modalité).

Le Groupe de Travail Chargé de la Révision précise que, dans l'état actuel de ses connaissances, DC:0-3R n'a pu ni fournir des critères détaillés pour les différents types de Troubles de la Régulation du traitement des stimuli sensoriels, ni spécifier le nombre de critères nécessaires pour faire le diagnostic. En lieu et place, DC:0-3R a voulu fournir une forme descriptive riche en critères, dans l'espoir que des recherches futures clarifieront ce domaine (DC:0-3R, p.12).

\section{C) Le traitement des stimuli sensoriels}

En mettant l'accent sur les patterns de la réactivité sensorielle, la nouvelle Classification attire l'attention sur le toucher, la vue, les bruits, le goût, l'odorat, les sensations de mouvements, les stimuli proprioceptifs et, sur les difficultés de l'enfant au niveau du traitement de ces stimuli sensoriels entrants, comme au niveau de la régulation des réponses qu'il apporte à ces stimuli. La Classification précédente attirait l'attention sur davantage de stimuli et de régulations en jeu, par exemple, la température, la douleur, la sensibilité buccale, la régulation de l'articulation des mots, de l'attention et de la concentration, du traitement de l'information auditivo-verbale, du traitement de l'information visuo-spatiale (DC:0-3, pp.41-44). Les observations cliniques et les recherches futures pourront être attentives à ces derniers aspects, d'autant qu'ils interviennent également dans les Troubles touchant de Multiples Domaines du Développement (MSDD). Or, pour certains enfants, il semble exister un continuum entre ces deux diagnostics (DC:0-3, p.128).

\section{D) Recherches futures}

Alors que le Groupe de Travail Chargé de la Révision (2005) et les cliniciens utilisateurs de la Classification ont apprécié l'utilité des Troubles de la Régulation, les experts ont cependant souligné la nécessité de recherches cliniques futures pour mieux identifier les catégories utiles pour le long terme (Fenichel, Emde, Egger et coll. 2005).

Pour une étude de cas cliniques nous recommandons la lecture du livre «Études de cas, Classification 0-3 ans » qui décrit remarquablement chaque situation clinique (Classification Diagnostique de 0 à 3 ans Études de cas, 2000).

Nous-mêmes, avec un petit groupe de cliniciens, nous poursuivons une recherche clinique sémiologique, réalisée avec un questionnaire rétroactif qui sera rempli par les parents ; ce questionnaire est lui-même le résultat d'une recherche préliminaire (Scholl, 2007). Cette étude - menée en population normale - veut établir, à partir d'une anamnèse parentale, une validation sémiologique développementale de 0 à 6 ans ; ensuite, les résultats seront comparés à une population d'enfants présentant des Troubles de la Régulation du traitement des stimuli sensoriels. 


\section{NOTRE EXPÉRIENCE}

\section{A) La Classification dans son ensemble}

La Classification 0-3 ans a été réalisée dans une grande ouverture d'esprit et elle apprend aux cliniciens à déployer des regards théoriques diversifiés tout en restant centrés sur l'observation et la réalité clinique. Elle se réfère aux théories du développement, à la psychologie du bébé, aux interactions précoces, aux théories de la relation et de l'attachement, à la psychodynamique, à la systémique familiale, à l'organisation par les bébés de leur expérience, aux modes de régulation et aux différences individuelles, au développement émotionnel, aux composantes constitutionnelles (corporelles) et aux tempéraments, aux théories de la régulation neurophysiologique, aux influences environnementales. Cette classification a le mérite d'avoir des fondements théoriques explicites qui peuvent être mis en cause par des découvertes scientifiques ; l'avancée des recherches cliniques permet donc de progressivement l'affiner. L'élaboration de cette classification a été réalisée à partir d'analyses de cas et en dialogue permanent avec des cliniciens et des parents qui ont pu l'amender. La classification révisée (DC:0-3R) nous semble plus précise que le DSM IV ou la CIM-10/ICD-10 ; elle observe des champs non parcourus par ces deux dernières. Elle porte attention aux signes dans un continuum du normal au pathologique, ce qui permet une identification du risque qui est tout aussi importante que celle du trouble pour l'enfant en bas âge et pour une action thérapeutique préventive. Le diagnostic de la classification "ne fige pas", il est une "photographie de l'instant présent" qui peut se répéter à plusieurs moments de la vie de l'enfant ; ce diagnostic est compris dans une évolution développementale. Les catégories sont davantage descriptives que nosographiques, ce qui convient à cet âge. Cette classification invite cependant l'intervenant à préciser un diagnostic aussi nuancé que possible. Pour le professionnel, la capacité à poser un profil diagnostique précis dans "l'ici et maintenant" peut faire prendre des décisions thérapeutiques qui amélioreront le pronostic. Dans ce sens, une classification diagnostique peut inciter à l'établissement d'un diagnostic plus précoce qui pourrait devenir un avantage pour l'enfant. Le diagnostic peut mieux faire percevoir plusieurs niveaux de difficultés qui nécessitent plusieurs abords thérapeutiques simultanés non perçus au départ. Le regard nuancé de cette classification invite à repérer au maximum les comorbidités, ce qui nuance d'autant plus l'évaluation. Dans notre expérience, le suivi thérapeutique n'est pas gêné par l'évaluation clinique de la classification, elle peut même représenter une aide en offrant un nouveau regard aux parents sur leur enfant. De plus, au vu de l'ensemble des cinq axes de la classification, l'établissement du diagnostic requiert l'engagement de l'évaluateur dans la relation. Le cinquième axe évalue "le niveau fonctionnel du développement émotionnel et social" de l'enfant dans sa relation au thérapeute. Répondre aux nuances de l'évaluation conduit le thérapeute à aiguiser son sens clinique avec la rigueur de l'observation. Nous constatons qu'au sein d'une réunion d'une équipe de travail pluridisciplinaire cet outil est un catalyseur d'échanges cliniques qui enrichit l'expérience et la réflexion. Elle favorise les échanges interdisciplinaires et l'apport mutuel des lectures complémentaires qui entrent en écho avec la classification. L'étendue de l'investigation clinique s'en trouve d'autant plus élargie. Lors de réunions avec d'autres professionnels tels que enseignants, puéricultrices,..., se référer aux lectures que donne la classification lors de l'observation de l'enfant permet de trouver un langage commun, un dialogue, un accord, et un nouveau regard sur l'enfant. Ce diagnostic - qui ne doit pas être explicitement formulé - est tout sauf une étiquette. Nous constatons aussi qu'il est un moyen d'échange avec des neuropédiatres ouvrant à un apport mutuel. Il est également un guide précieux dans l'évaluation de l'évolution de l'enfant. En psychothérapie, il peut objectiver des changements (positifs) après quelques mois de prise en charge. Notons enfin que la Classification 0-3 ans 
est la première classification diagnostique qui donne une description qualitative du trouble de la relation (axe II). On n'y trouve cependant pas d'attributions positives de l'interaction ce qui pourrait être l'objet de révisions futures.

\section{B) Les Troubles de la Régulation}

Dans notre expérience, la révision de la Classification a nettement amélioré la précision clinique et la validité du diagnostic de Troubles de la Régulation du traitement des stimuli sensoriels. Cependant, comme nous l'avons déjà indiqué, il nous semble que certains enfants peuvent présenter simultanément deux des trois modalités, particulièrement la modalité "Recherchant des stimulations sensorielles et/ou Impulsif" avec la modalité Hypersensible. Nous voudrions proposer la possibilité de faire le diagnostic de plusieurs modalités simultanément en introduisant une cotation en gradient d'intensité pour chacune d'elles. Nous constatons que le diagnostic de Troubles de la Régulation du traitement des stimuli sensoriels peut être utilisé jusqu'à l'âge de 6 ans. Dans ce cas, une anamnèse rétroactive rapportant le décours développemental symptomatique permet de nuancer l'expressivité des signes. Ceci invite à un travail de recherche déterminant l'équivalence des symptômes dans un décours évolutif en fonction de l'âge. Des comparaisons à une population témoin permettraient de mieux déterminer la sémiologie : les signes les plus pertinents, les variations par rapport à la normale, les variations de la réactivité, des critères quantitatifs de sévérité. On pourrait rechercher à mieux différencier entre eux : les troubles de la régulation sensorielle, les difficultés de régulation émotionnelle et les régulations de l'attention. Alors que la forme révisée l'omet, une recherche sémiologique pourrait mieux déterminer la présence simultanée de difficultés au niveau du traitement de l'information visuo-spatiale, auditivo-verbale et de régulations au niveau de la sphère buccale. Une corrélation avec la clinique du sommeil pourrait être recherchée et précisée. Ceci invite à réaliser un travail pluridisciplinaire, avec des regards théoriques diversifiés, afin de rechercher des corrélations sémiologiques dans des sphères différentes et en précisant toujours un décours développemental. En plus du "Profil sensoriel" (Dunn, 1999) qui évalue les régulations sensorielles, d'autres outils de mesure devraient être créés afin d'évaluer d'autres polarités sémiologiques des troubles de la régulation. Cette sémiologie devrait être évaluée dans un continuum du normal au pathologique afin de mieux déterminer différents gradients d'intensité et de préciser une marge indicatrice pour une intervention préventive. La recherche de comorbidités permettrait de mieux déterminer la possibilité que certaines pathologies existantes n'excluent pas la présence simultanée de troubles de la régulation. Nous constatons entre autres cette association avec des troubles de l'affect.

Dans notre expérience clinique, la notion de troubles de la régulation - en lien avec les notions de régulations neurophysiologiques, de tempérament et de constitution - nous a invité à mieux préciser la part de fonctionnement propre de l'enfant au sein de difficultés relationnelles parentales. Dans le processus psychothérapeutique et dès le bilan initial, nous cherchons à réaliser un discernement - dans un continuum - des parts respectives : constitutionnelle, psychologique et relationnelle. Cela nous invite à être plus nuancé dans nos interventions en restituant ces parts respectives dans la perception que nous proposons. Il en résulte souvent une meilleure compréhension de l'enfant par les parents dont la déculpabilisation leur permet d'exprimer plus ouvertement leurs sentiments contretransférentiels et leurs difficultés affectives. De même, les Troubles touchant de Multiples Domaines du Développement (MSDD) comprennent souvent des troubles importants de la régulation. Apporter aux parents un regard nouveau sur l'enfant à partir d'une compréhension davantage neurophysiologique permet de donner un sens à des difficultés relationnelles induites par l'enfant, de faire sortir de la confusion les parents et d'ouvrir à la 
relation. Ce même regard permet également de souligner des compétences de l'enfant. Ceci donne à considérer le spectre autistique dans un décours évolutif positif et réellement constaté en psychothérapie.

Pour conclure, la clinique des Troubles de la Régulation du traitement des stimuli sensoriels et les recherches s'y rapportant sont à la convergence de l'intérêt tant des pédopsychiatres, psychologues, psychomotriciens, ergothérapeutes, thérapeutes du développement, que des neuropédiatres confrontés à des enfants de 0 à 5 ans; de plus, cliniques et recherches en ce domaine peuvent enrichir la pluridisciplinarité et les pratiques de la psychothérapie.

Résumé. En 1994 est parue la première «Classification Diagnostique des Troubles de la Santé Mentale et du Développement de la Petite Enfance »et, en août 2005, l'Édition Révisée (DC:0-3R). Cet article présente la révision des critères du diagnostic: Trouble de la Régulation du traitement des stimuli sensoriels. Ce diagnostic, innovateur, comprend trois modalités: Hypersensible, Hyposensible/Sous-réactif, et Recherchant des stimulations sensorielles et/ou Impulsif. Ces enfants ont des difficultés au niveau du traitement des stimuli sensoriels entrants (le toucher, la vue, les bruits, le goût, l'odorat, les sensations de mouvement) et au niveau de la régulation des réponses qu'ils apportent à ces stimuli. Ce diagnostic se réfère à leurs difficultés à réguler leurs émotions, leurs comportements et leurs habilités motrices en réponse aux stimulations sensorielles.

Summary. In 1994 was published the first « Diagnostic Classification of Mental Health and Developmental Disorders of Infancy and Early Childhood », and in August 2005 the Revised Edition » (DC:0-3R). This article presents the revised criteria of the diagnosis: Regulation Disorders of Sensory Processing. This innovative diagnosis includes three types: Hypersensitive, Hyposensitive/Underresponsive and Sensory Stimulation-Seeking/Impulsive. These children have difficulty processing sensory input (touch, sight, sound, taste, smelt, sensation of movement) and regulating their responses. This diagnosis refers to children's difficulties in regulating emotions, behaviours and motor abilities in response to sensory stimulation.

Mots-clés. Trouble de la Régulation - Diagnostic - Nourrisson.

Key words. Regulation Disorders - Diagnosis - Infant.

\section{References}

Ayres J. (1972): Sensory integration and learning disabilities. Western Psychological Services, Los Angeles. In Williamson G.G., Anzalone M.E.: Sensory Integration and Self-Regulation in Infants and Toddlers: Helping Very Young Children Interact With Their Environment, Zero to Three, Washington DC, 2001.

Chess S., Thomas A. (1986): Temperament in clinical practice. Guildford Press, New York.

Cordeiro M.J., Caldeira da Silva P. (1998): La classification diagnostique des troubles de la santé mentale du nourrisson. Une expérience clinique. Devenir, 10, 51-65.

DC: 0-3 Casebook, by Zero to three/National Center for Infant, Toddlers and Families, Washington, DC, 1997. Trad. fr. Classification Diagnostique de 0 à 3 ans Études de cas, Médecine et Hygiène, Genève, 2000.

DeGangi G.A., DiPietro J.A., Greenspan S.I., Porges S.W. (1991): Psychophysiological characteristics of the regulatory disordered infant. Infant Behavior \& Development, 14: 37-50.

DeGangi G.A., Porges S.W., Sickel R.Z., Greenspan S.I. (1993). Four-year follow-up of a sample of regulatory disordered infants. Infant Mental Health Journal, 14, 4: 330-343.

DeGangi G.A., Poisson S., Sickel R.Z., Wiener A.S. (1995): The Infant-Toddler Symptom Checklist. Therapy Skill Builders, Tucson, Arizona.

DeGangi G.A., Balzer-Martin L.A. (1999): The sensorimotor history questionnaire for preschoolers. Journal of Developmental and Learning Disorders, 3, 1: 59-83. 
DeGangi G.A., Breinbauer C., Roosevelt J.D., Porges S.W., Greenspan S. (2000): Prediction of childhood problems at three years in children experiencing disorders of regulation during infancy. Infant Mental Health Journal, 3, 21: 156-175.

Dunn W. (1999): The Sensory Profile manual. Psychological Corporation, San Antonio TX.

Fenichel E., Emde R.N., Egger H., Guedeney A., Wise B.K., Wright H.H. (2005): Clinique Présentation de la révision de la Classification Diagnostique 0-3 ans (DC:0-3R). Devenir, 17, 4: 347-360.

Greenspan S. (1992): Infancy and early childhood: The practice of clinical assessment and intervention with emotional and developmental challenges. International Universities Press, Madison CT.

Greenspan S., Wieder S. (1993): Regulatory disorders. In Zeanah: Handbook of infant mental health, (pp.280290), The Guilford Press, New York.

Guedeney N., Guedeney A., Danon G., Mintz A.S., Morales-Huet M., Rabouam C., Jacquemain F., Le Nestour A., Roujean S. (2002): À propos des systèmes de classification en psychiatrie du très jeune enfant : utilisation de la classification diagnostique Zero to three. Psychiatrie de l'enfant, 45, 2: 483-531.

Rothbart M.K., Chew K.H., Gartstein M.A. (2001): Assessment of temperament in early development. In Singer L.T. \& Zeskind P.S., Biobehavioral assessment of the infant (pp.190-208), Guilford Press, New York.

Scholl J-M. (2007): Classification diagnostique 0-3 ans: recherche sémiologique sur les troubles de la régulation. Psychiatrie de l'enfant (in press).

Skovgaard A.M., Houmann T., Landorph S.L.,Christiansen E. (2004): Assessment and classification of psychopathology in epidemiological research of children 0-3 years of age. A review of the literature. European Child \& Adolescent Psychiatry, 13, 6: 393-346.

Skovgaard A.M., Houmann T., Christiansen E., Andreasen A.H. (2005): The reliability of the ICD 10 and the DC 0-3 in an epidemiological sample of children $1 \frac{1}{2}$ years of age. Infant Mental Health Journal, 26, 5: 470480.

Sturm L. (2004): Temperament in Early Childhood: A Primer for the Perplexed. Zero to Three, mars 2004: 4-11.

Thomas J.M., Harmon R.J. (1998): La classification diagnostique des troubles de la santé mentale et du développement de la première et de la petite enfance. Un système dynamique pour la connaissance et le traitement des nourrissons, des jeunes enfants et de leurs familles. Devenir, 10, 1: 35-50.

Williamson G.G., Anzalone M.E. (2001): Sensory Integration and Self-Regulation in Infants and Toddlers: Helping Very Young Children Interact With Their Environment. Zero to Three, Washington DC.

ZERO TO THREE: Diagnostic Classification of Mental Health and Developmental Disorders of Infancy and Early Childhood (DC:0-3), Washington, DC, 1994. Trad. fr. Classification Diagnostique de 0 à 3 ans, Médecine et Hygiène, Genève, 1998.

ZERO TO THREE: Diagnostic Classification of Mental Health and Developmental Disorders of Infancy and Early Childhood (DC:0-3R), Revised Edition, ZERO TO THREE Press, Washington, DC, 2005. 